\title{
A Comparison of Inpatient Cost Per Day in General Surgery Patients with Type 2 Diabetes Treated with Basal-Bolus versus Sliding Scale Insulin Regimens
}

\author{
Victoria L. Phillips ${ }^{1} \cdot$ Anwar L. Byrd $^{2} \cdot$ Saira Adeel $^{2} \cdot$ Limin Peng $^{3}$ • \\ Dawn D. Smiley ${ }^{2} \cdot$ Guillermo E. Umpierrez $^{2}$
}

Published online: 21 April 2017

(c) The Author(s) 2017. This article is an open access publication

\begin{abstract}
Background The identification of cost-effective glycaemic management strategies is critical to hospitals. Treatment with a basal-bolus insulin (BBI) regimen has been shown to result in better glycaemic control and fewer complications than sliding scale regular insulin (SSI) in general surgery patients with type 2 diabetes mellitus (T2DM), but the effect on costs is unknown.

Objective We conducted a post hoc analysis of the RABBIT Surgery trial to examine whether total inpatient costs per day for general surgery patients with T2DM treated with BBI $(n=103)$ differed from those for patients with T2DM treated with SSI $(n=99)$ regimens.

Methods Data were collected from patient clinical and hospital billing records. Charges were adjusted to reflect hospital costs. General linearized models were used to estimate the risk-adjusted effects of BBI versus SSI treatment on average total inpatient costs per day.

Results Risk-adjusted average total inpatient costs per day were \$US5404. Treatment with BBI compared with SSI reduced average total inpatient costs per day by $\$$ US751 (14\%; 95\% confidence interval [CI] 20-4). Being treated in a university medical centre, being African American or
\end{abstract}

Victoria L. Phillips

vphi101@sph.emory.edu

1 Department of Health Policy and Management, Rollins School of Public Health of Emory University, 1518 Clifton Road, Atlanta, GA 30322, USA

2 Department of Medicine, Division of Endocrinology, Emory University School of Medicine, Atlanta, GA, USA

3 Department of Biostatistics, Rollins School of Public Health, Atlanta, GA, USA having a bowel procedure or higher-volume pharmacy use significantly reduced costs per day.

Conclusions In general surgery patients with T2DM, a BBI regimen significantly reduced average total hospital costs per day compared with an SSI regimen. BBI has been shown to improve outcomes in a randomized controlled trial. Those results, combined with our findings regarding savings, suggest that hospitals should consider adopting BBI regimens in patients with T2DM undergoing surgery.

\section{Key Points for Decision Makers}

This is the first study to show that a basal-bolus insulin regime compared with sliding scale insulin reduces total hospital costs per day by $14 \%$ among general surgery patients with type 2 diabetes mellitus.

These findings, combined with data from the randomized controlled trial showing that basal-bolus insulin improves outcomes, suggest that hospitals should consider the adoption of BBI.

\section{Introduction}

Diabetes mellitus is a common, serious and expensive condition that incurred costs estimated at \$US245 million in the USA in 2013, with hospital expenditures accounting for $43 \%$ of the total [1]. Observational studies and randomized controlled trials (RCTs) have documented that hyperglycaemia and diabetes are associated with poor outcomes in hospital patients [2,3], including increased 
rates of multi-organ system failure [4], infection [5, 6] and mortality [7-9]. Glycaemic control management programmes have been shown to reduce the occurrence of these events [10] and hospital costs in both critical care and cardiac patients [4, 11-13]. However, data regarding the impact that improved glycaemic control can have on costs for general surgical patients with type 2 diabetes mellitus (T2DM) in non-intensive care unit (ICU) settings who have undergone a wide array of procedures are very limited.

Changes in the payment policies of Medicare, the US insurer for people aged $\geq 65$ years, mean hospitals face significant penalties of amounts up to $3 \%$ of all Medicare payments if infection or readmission rates for five conditions exceed the risk-adjusted projected rates for the hospital [14]. As patients with diabetes are at increased risk for both, improved clinical management of this group is increasingly important [15].

Furthermore, Medicare is moving toward bundled or fixed payments for a care episode, which means reimbursement will be determined by diagnosis rather than actual resources used by a particular patient during a hospital stay [16]. This payment system places hospitals at increased financial risk for patients with more expensive conditions [17]. In this environment, patients with poorly managed diabetes present a substantial liability as they are known to generally have more costly inpatient stays than those without the condition [18].

The RABBIT 2 Surgery trial was a multicentre RCT that investigated a basil-bolus insulin (BBI) regimen compared with a sliding scale insulin (SSI) regimen for managing T2DM in adults ( $n=211$ ) undergoing general (non-cardiac) surgery. The study was a 2-year study initiated in December 2007 and followed patients during a single hospitalization or an index hospital stay at which they were enrolled in the trial [19]. The trial was powered on its primary outcome measures: daily blood glucose levels and a composite of postoperative complications, including wound infections.

Patients were randomly assigned to receive either (1) BBI with glargine once daily and glulisine before meals or (2) the standard regimen of SSI administration four times daily for a blood glucose level of $>140 \mathrm{mg} / \mathrm{dl}$. The timeframe for the intervention was a single or index hospitalization, meaning the hospitalization at which they were enrolled in the trial. The trial is noteworthy as participants had a wider range of diagnoses than earlier studies.

The study found that patients treated with BBI had significantly better post-surgery glycaemic control (144.9 vs. $174.2 \mathrm{mg} / \mathrm{dl}$ ), fewer wound infections (2.9 vs. 10.3\%) and a lower percentage of post-operative complications (8.6 vs. $24 \%$ ) than those treated with SSI $[10,19]$. Current clinical guidelines now recommend the use of BBI in general surgery patients specifically to help manage diabetes and hyperglycaemia [20,21].
Given the improvement in clinical outcomes with the use of BBI, financial data for participants in the RABBIT 2 Surgery trial were gathered and combined with demographic and clinical information for analysis to determine whether BBI compared with SSI treatment translated into inpatient hospital savings.

The study was a cost analysis conducted from the hospital perspective, and financial data were gathered for length of hospital stay (LOS) for each participant. Given the improved outcomes documented in the RABBIT 2 Surgery trial, should BBI prove to be cost neutral or to generate cost savings, it would be a dominant strategy [22] The Institutional Review Board at Emory University approved this study.

\section{Methods}

\subsection{Data Sources and Elements}

Clinical data were retrieved from patients' electronic medical records. Data on hospital costs and service utilization for pharmacy were obtained from the billing departments at the two participating institutions: (1) Emory University Hospital, a tertiary care university medical centre linked with a medical school and involved in the training of physicians and (2) Grady Memorial Hospital, a public community teaching hospital and level 1 trauma centre. Both are located in Atlanta, GA, USA.

Data extracted from the medical records included diagnoses based on the International Statistical Classification of Diseases and Related Health Problems (ICD-9), Current Procedural Terminology (CPT) codes and Medicare Severity diagnosis-related group (MS-DRG) codes, which form the basis of hospital billing to insurers [23]. Information was also collected on age, race, sex, treatment facility and inpatient LOS. Patient baseline health status was measured through the American Heart Association Classification of Functional Capacity and Objective Assessment (AHA score), commonly used in hospitals [24].

Patients were admitted under 109 different DRGs. Although the DRG system is run by the Medicare programme, all patient admissions are classified using DRGs regardless of whether the patient is insured by Medicare. To further capture variations in patient health status, we created variables for whether or not patients were classified under the two most common DRGs: (1) DRGs 329-331, representing major and minor bowel procedures $(n=20)$ and (2) DRGs 406-407, representing pancreas, liver and shunt procedures $(n=26)$. In addition, mean number of pharmacy units consumed was also included as a proxy measure of patient health status. 
ICD-9 data were missing for over $10 \%$ of patients, so they were not used in the calculations because of the impact on sample size and because they may not have been missing in a random fashion.

Total inpatient charges during the index hospitalization were collected for each patient. Charges represent the amounts billed by hospitals for each service provided. They do not represent the amounts reimbursed by payers or the actual costs to the hospital of providing the service. Rather, charges are what the hospitals bill insurers for the cost of the hospital stay [25].

Per cost-effectiveness study recommendations, charges were converted to costs using Medicare cost-to-charge ratios [26]. These ratios are derived from cost reports submitted by hospitals to the Center for Medicare and Medicaid Services (CMS) to help the agency establish reimbursement rates [27]. We applied the institutionspecific Medicare cost-to-charge ratio as found in patient billing records. Thus, we refer to hospital costs in our evaluation. All costs were calculated in \$US and converted from year 2011 values by adjusting for inflation using standard methods. This resulted in a $19 \%$ price increase to allow for reporting in year 2015 values $^{1}$ [28].

\subsection{Statistical Methods}

The dependent variable in the model was average total inpatient costs per day, calculated as total inpatient costs/ LOS. Model covariates comprised the treatment group and several measures of patient health status, including the AHA severity score, whether or not the patient underwent bowel or pancreatic and liver procedures, and utilization of pharmacy items.

Other covariates were age, race, sex and site of care. The latter was included for two reasons: (1) although charges were converted to costs at each institution using the costto-charge ratio, the base cost structure is likely to differ between facilities given their distinct types; (2) physician care practices may differ at each facility, which may impact on costs through effects on service utilization and $\operatorname{mix}[29]$.

We used non-parametric Wilcoxon tests (or KruskalWallis tests) for continuous variables and Chi squared tests (or Fisher's exact test) for categorical variables to compare base values by treatment group. As recommended for small cost datasets, generalized linear models (GLMs), specified as Gamma distributions with a log-link function, were used to identify the effect of BBI treatment on costs and utilization [30]. The $\beta$ coefficients on the control variables, were exponentiated, $E[y \mid x . z] \mid=\hat{y}=\exp (\beta)$, where $y$ equals

\footnotetext{
$\overline{1^{1} \text { Value }(\$)_{\mathrm{BY}}}=$ Value $\left.(\$)_{\mathrm{PY}}\left[\mathrm{CPI}_{\mathrm{BY}} / \mathrm{CPI}_{\mathrm{PY}}\right)\right]$ where $B Y$ base year, $P Y$ past year, $C P I$ consumer price index.
}

costs or use, $x$ equals treatment group and $z$ equals control variables, to allow interpretation of the coefficient as multiplicative effects. Model fit was assessed by the squared deviance divided by degrees of freedom, and $1.00 \pm 0.13$ was viewed as an acceptable range [31, 32] Statistical analyses were performed using SPSS (v23) [33]. A $p$ value $\leq 0.05$ was considered significant.

\section{Results}

Of the 211 patients in the RABBIT Surgery trial, 202 were included in this cost analysis (99 in the BBI group and 103 in the SSI group). Five patients were excluded because of incomplete financial data, and four were treated by another facility. Table 1 provides data on a range of patient characteristics, including blood glucose levels, treatment location, costs and utilization rates. Significant differences between the treatment groups are shown in the last column of the table.

As expected, based on their randomization in the RABBIT 2 trial, the two groups were well matched in terms of demographic and clinical factors. The mean age of participants was 58.5 years, half of the sample was female and 55\% were African American. Mean total hospital costs were \$US29,104 and mean LOS was 9 days. Neither differed significantly between the treatment groups.

Table 2 shows the multivariate results for average total hospital costs per day. Estimated total inpatient costs per day were \$US5404. Treating patients with BBI versus SSI reduced costs per day by \$US771 [14\%; 95\% confidence interval (CI) 20-4]. Receiving treatment at a university medical centre as against a public hospital reduced costs by $22 \%$ for a savings of \$US1190 per day. Being African American reduced average total costs per day by $44 \%$, having a DRG related to bowel procedures by $33 \%$, and having a relatively high volume of pharmacy use by $1 \%$, a very small effect size. All values were significant at $p \leq 0.05$.

\section{Discussion}

The results of this cost analysis show that treatment with a BBI compared with an SSI regimen in general surgery patients with T2DM lowered the average total inpatient hospital costs per day by $14 \%$. This finding is significant given results from the RABBIT 2 trial found that BBI improves outcomes. This, combined with the results that show BBI regimens also reduce costs, indicate that hospitals should consider adopting the BBI approach. This is notable, because prior studies have focused on patients with similar conditions, such as cardiac ailments, or being 
Table 1 Patient characteristics, location, cost and utilization by the full sample and comparisons between treatment groups

\begin{tabular}{|c|c|c|c|c|}
\hline Characteristics & Full sample & Basal-bolus regimen & Sliding scale regimen & $p$ values ${ }^{\mathrm{a}}$ \\
\hline Patients $(n)$ & 202 & 99 & 103 & \\
\hline Age, (years) & $58.5 \pm 11.4$ & $58.9 \pm 12.7$ & $58.1 \pm 0.20$ & 0.612 \\
\hline Female sex & $104(50)$ & $50(50)$ & $54(51)$ & 0.892 \\
\hline African American race & $113(55)$ & $55(55)$ & $58(55)$ & 0.518 \\
\hline Admission blood glucose, $\mathrm{mg} / \mathrm{dl}(n \pm \mathrm{SD})$ & $186.7 \pm 90.2$ & $192.2 \pm 102.4$ & $181.4 \pm 77.1$ & 0.68 \\
\hline Randomization blood glucose, $\mathrm{mg} / \mathrm{dl}(n \pm \mathrm{SD})$ & $197.5 \pm 54.7$ & $198.9 \pm 50.8$ & $196.1 \pm 58.5$ & 0.49 \\
\hline Blood glucose after surgery, mg/dl $(n \pm \mathrm{SD})$ & $159.9 \pm 43.6$ & $144.9 \pm 31.7$ & $174.2 \pm 48.5$ & $<0.001$ \\
\hline Treated at University Medical Center & $102(50)$ & 49 (49) & $52(50)$ & 0.498 \\
\hline $\begin{array}{l}\text { American Heart Association Classification of } \\
\text { Functional Capacity score }\end{array}$ & $1.61 \pm 0.56$ & $1.61 \pm 0.58$ & $1.63 \pm 0.54$ & 0.776 \\
\hline Total inpatient costs ${ }^{\mathrm{b}, \mathrm{c}}$ & $24457 \pm 18359$ & $23226 \pm 18745$ & $25641 \pm 17991$ & 0.132 \\
\hline Length of stay (days) & $9.00 \pm 7.79$ & $8.97 \pm 8.60$ & $9.0 \pm 6.97$ & 0.957 \\
\hline Inpatient costs per day ${ }^{\mathrm{d}}$ & $4541 \pm 18359$ & $3907 \pm 6606$ & $3724 \pm 4020$ & 0.813 \\
\hline Intensive care unit room costs & $468 \pm 2083$ & $432 \pm 2491$ & $462 \pm 1487$ & 0.916 \\
\hline Days in intensive care unit & $0.38(1.93)$ & $0.25(0.88)$ & $0.51(2.61)$ & 0.342 \\
\hline Used intensive care unit & $26(13)$ & $11(11)$ & $25(14)$ & 0.341 \\
\hline Number of pharmacy prescriptions & $187 \pm 182$ & $173 \pm 167$ & $203 \pm 196$ & 0.247 \\
\hline \multicolumn{5}{|l|}{ Primary diagnosis-related group } \\
\hline Major and minor bowel procedures $(\%)$ & 13.7 & 14.4 & 13.0 & 0.467 \\
\hline Pancreas, liver and shunt procedures $(\%)$ & 9.9 & 7.6 & 12.1 & 0.280 \\
\hline
\end{tabular}

Data are presented as mean \pm standard deviation (SD) or $n(\%)$ unless otherwise specified. All costs are presented as $\$ \mathrm{US}^{\mathrm{b}} . \mathrm{mg} / \mathrm{dl}$ micrograms per decilitre

$B Y$ base year, $C P I$ consumer price index, $P Y$ past year, $S D$ standard deviation

${ }^{\text {a }} p$ values refer to comparisons between treatment groups

b All costs converted from year 2011 values to year 2015 values based on the following formula: Value $(\$ U S)_{\mathrm{BY}}=$ Value $\left.(\$ U S)_{P Y}\left[\mathrm{CPI}_{\mathrm{BY}} / \mathrm{CPI}_{\mathrm{PY}}\right)\right]$ where

c Converted total mean inpatient costs from \$US24457 to \$US29103

${ }^{\mathrm{d}}$ Converted cost per day from \$US5409 to \$US5404

Table 2 Multivariate analysis of the mean total inpatient cost per day

\begin{tabular}{llll}
\hline Covariates & $\beta$ Coefficent $(95 \%$ CI $)$ & $p$ value & Exp $\beta$ \\
\hline Intercept & $8.599(8.146-9.053)$ & 0.000 & $4541^{\text {a }}$ \\
Basal bolus insulin regime & $-0.152(-0.299$ to 0.005$)$ & 0.043 & 0.86 \\
University medical centre location & $-0.247(-0.439$ to -0.059$)$ & 0.010 & 0.78 \\
Age & $0.003(-0.004$ to 0.010$)$ & 0.421 & NS \\
African American race & $-0.417(-0.578$ to -0.256$)$ & 0.000 & 0.65 \\
Female sex & $-0.051(-0.214$ to 0.111$)$ & 0.536 & NS \\
American Heart Association score & $0.008(-0.132$ to 0.148$)$ & 0.911 & NS \\
Bowel-related diagnosis-related group & $-0.263(-0.499$ to -0.027$)$ & 0.029 & 0.77 \\
Pancreas-related diagnosis-related group & $-0.179(-0.437$ to 0.070$)$ & 0.174 & NS \\
Level of pharmacy utilization & $-0.001(-0.0012$ to -0.008$)$ & 0.000 & 0.0008 \\
\hline
\end{tabular}

CI confidence interval, $N S$ not significant

${ }^{a}$ Reported in the text in \$US, year 2015 values: \$US5404 treated in specific settings such as an ICU rather than in a generalized setting.

Our findings are in accord with other analyses of glycaemic management programmes in that we found decreased costs $[12,13]$. These interventions are very inexpensive, which is critical; one study reported net savings of \$US1885 per day from an ICU management programme [11]. Overall inpatient average cost estimates 
reported here are also similar to those for the state of Georgia, USA, in 2014 (\$US1588) [34]. While many factors produce the differences in these values, one is our focus on patients with diabetes.

Receiving care at a university medical centre rather than a public hospital reduced overall costs by $22 \%$. This facility effect may be attributable to a number of factors. Differences in the base cost structure likely exist between facilities. Grady Memorial Hospital, a teaching hospital, level 1 trauma centre is likely to have a higher cost base than Emory University Hospital. Differences in care practices may have also affected care pathways, ultimately resulting in differences in use and costs of resources. Together, these factors strongly suggest that controlling for multiple facility effects, where applicable, is warranted and is an important area for further investigation.

Regarding other covariates, age and sex had no impact on average total costs per day, while being African American reduced them substantially. This latter effect may be partly due to differences in the disease profiles between African American and White populations occurring during the study time period. These may not have been fully captured by the available health status measures. More African Americans were treated at Grady Memorial Hospital, where care practices may differ from those at Emory University Hospital and but not be fully captured by the facility variables. This area warrants further exploration.

Two health status measures were significant: having a bowel procedure and the volume of pharmacy utilization. The results indicate that bowel procedures are relatively less expensive than procedures under different DRGs, including diseases related to cancer, heart procedures, stroke and amputations. Increased pharmacy utilization reduced overall costs, but the effect was very small. This suggests that pharmacy use may impact costs by improving patient health status and thereby reducing overall costs. Data on the items used are needed for further exploration of this relationship.

Adopting a BBI regimen and thereby reducing costs would also potentially help hospitals adapt to new Medicare payment policies. The RABBIT 2 Surgery trial found BBI reduced complications, including hospital-acquired infections and pneumonia, two conditions for which Medicare is fining hospitals if they occur above the expected risk-adjusted rate $[10,16]$. Furthermore, as BBI lowers costs for patients with diabetes, hospital financial risks under bundled payment systems are likely reduced. Medicare is levying penalties for readmissions within 30 days for select conditions. Data were not available on readmission rates, but this is an important area for further research.

Our findings reflect the limitations of the RABBIT 2 Surgery trial. The sample size is small and represents patients from two distinct urban medical centres located in the south of the USA. Thus, these results may not be generalizable to general hospitals with a different patient mix, mission, staff make-up or cost structure. Participants had been diagnosed with diabetes and were under the care of a physician. Undiagnosed cases may have a different result. Also, our measures of symptom severity may not have fully captured the underlying health status of the patient. Furthermore, missing data precluded including specific diagnoses in the analysis. These results should be confirmed in a larger, more diverse sample.

\section{Conclusions}

In spite of the limitations, our results strongly suggest that a BBI glycaemic management strategy is worth pursing because it may reduce costs. Work has also documented that it improves a range of outcomes in a general surgery population. Use of a BBI regimen may also help hospitals reduce infection costs, and thereby avoid current Medicare penalties, and reduce patient costs, which is a key goal should fixed per-patient payments become the norm.

Acknowledgements Dr Umpierrez is supported in part by research grants from the American Diabetes Association (7-03-CR-35) and PHS Grant UL1 RR025008 from the Clinical and Translational Science Award Program (M01 RR-00039), National Institutes of Health, National Center for Research Resources. He has received grant support (to Emory University) from Merck, Sanofi, Boehringer Ingelheim, Astra Zeneca and Novo Nordisk.

Author contributions Proposal authors: DDS, GEU, VLP. Obtaining funding: DS, GEU. Study design: VP, DS, GEU. Cost-analysis design: VLP, DDS. Data collection: ALB, DDS, SA. Data analysis: VLP, LP. Manuscript preparation: VLP, GEU. Data acquisition and interpretation: SA, AB, VP, DS, GEU.

\section{Compliance with Ethical Standards}

This study is adjunct to the RABBIT 2 Surgery trial (ClinicalTrials.gov identification: NCT00596687) [10]. Pilot data from this study were presented in abstract form at the 73rd Scientific Sessions of the American Diabetes Association, Chicago, IL. The Emory University Institutional Review Board (IRB) approved this study and informed consent was obtained for all patients in accordance with IRB guidelines.

Conflict of interest Victoria L Phillips, Anwar L Byrd, Saira Adeel, Limin Peng, Dawn D Smiley and Guillermo E Umpierrez, have no conflicts of interest, financial or otherwise, in relation to the manuscript.

Funding This investigator-initiated study was supported by an unrestricted grant from Sanofi (Bridgewater, NJ, USA). The sponsor had no input into the results presented here.

Data Availability Statement The dataset contains complete medical records for the sample. Upon request, the authors will provide a deidentified dataset. Given the nature of the data, the Institutional 
Review Board require the authors to catalogue researchers accessing the data.

Open Access This article is distributed under the terms of the Creative Commons Attribution-NonCommercial 4.0 International License (http://creativecommons.org/licenses/by-nc/4.0/), which permits any noncommercial use, distribution, and reproduction in any medium, provided you give appropriate credit to the original author(s) and the source, provide a link to the Creative Commons license, and indicate if changes were made.

\section{References}

1. American Diabetes Association. The cost of diabetes. http:// www.diabetes.org/advocacy/news-events/cost-of-diabetes.html. Accessed 23 July 2015.

2. Baker EH, Janaway CH, Philips BJ, Brennan AL, Baines DL, Wood DM, Jones PW. Hyperglycaemia is associated with poor outcomes in patients admitted to hospital with acute exacerbations of chronic obstructive pulmonary disease. Thorax. 2006;61(4):284-9.

3. Estrada CA, Young JA, Nifong LW, Chitwood WR. Outcomes and perioperative hyperglycemia in patients with or without diabetes mellitus undergoing coronary artery bypass grafting. Ann Thorac Surg. 2003;75(5):1392-9.

4. Van den Berghe G, Wouters PJ, Kesteloot K, Hilleman DE. Analysis of healthcare resource utilization with intensive insulin therapy in critically ill patients. Crit Care Med. 2006;34(3):612-6.

5. Furnary AP, Zerr KJ, Grunkemeier GL, Starr A. Continuous intravenous insulin infusion reduces the incidence of deep sternal wound infection in diabetic patients after cardiac surgical procedures. Ann Thorac Surg. 1999;67(2):352-60 (discussion 360-352).

6. Pomposelli JJ, Baxter JK 3rd, Babineau TJ, et al. Early postoperative glucose control predicts nosocomial infection rate in diabetic patients. JPEN J Parenter Enteral Nutr. 1998;22(2):77-81.

7. Kosiborod M, Rathore SS, Inzucchi SE, Masoudi FA, Wang Y, Havranek EP, Krumholz HM. Admission glucose and mortality in elderly patients hospitalized with acute myocardial infarction: implications for patients with and without recognized diabetes. Circulation. 2005;111(23):3078-86.

8. Falciglia M, Freyberg RW, Almenoff PL, D'Alessio DA, Render ML. Hyperglycemia-related mortality in critically ill patients varies with admission diagnosis. Crit Care Med. 2009;37(12):3001-9.

9. Umpierrez GE, Isaacs SD, Bazargan N, You X, Thaler LM, Kitabchi AE. Hyperglycemia: an independent marker of in-hospital mortality in patients with undiagnosed diabetes. J Clin Endocrinol Metab. 2002;87(3):978-82.

10. Umpierrez GE, Smiley D, Jacobs S, et al. Randomized study of basal-bolus insulin therapy in the inpatient management of patients with type 2 diabetes undergoing general surgery (RABBIT 2 surgery). Diabetes Care. 2011;34(2):256-61.

11. Krinsley JS, Jones RL. Cost analysis of intensive glycemic control in critically ill adult patients. Chest. 2006;129(3):644-50.

12. Newton CA, Young S. Financial implications of glycemic control: results of an inpatient diabetes management program. Endocr Pract. 2006;12(Suppl 3):43-8.

13. Sadhu AR, Ang AC, Ingram-Drake LA, Martinez DS, Hsueh WA, Ettner SL. Economic benefits of intensive insulin therapy in critically Ill patients: the targeted insulin therapy to improve hospital outcomes (TRIUMPH) project. Diabetes Care. 2008;31(8):1556-61
14. Centers for Medicare \& Medicaid Services. Readmissions reduction program. http://www.cms.gov/Medicare/Medicare-Feefor-Service-Payment/AcuteInpatientPPS/Readmissions-ReductionProgram.html. Accessed 23 July 2015.

15. Thomas RT. Diabetes mellitus and cardiothoracic surgical site infections. Am J Infect Control. 2005;33(6):353-9.

16. Association of American Medical Colleges. Selected Medicare hospital quality provisions under the ACA. http://www.aamc.org/ advocacy/medicare/154226/quality_regulations.html. Accessed 23 July 2015.

17. Centers for Medicare \& Medicaid Services. Bundled payments for care improvement (BPCI) initiative: general information. https://innovation.cms.gov/initiatives/bundled-payments. Accessed 3 Oct 2016.

18. Florentino C, Gabriel O, Jose S, Lourdes G, Alvaro S, Manuel A. Care resource utilization and direct costs incurred by people with diabetes in a Spanish hospital. Diabetes Res Clin Pract. 2002;56(1):27-34.

19. Umpierrez GE, Smiley D, Zisman A, et al. Randomized study of basal-bolus insulin therapy in the inpatient management of patients with type 2 diabetes (RABBIT 2 trial). Diabetes Care. 2007;30(9):2181-6.

20. Umpierrez GE, Hellman R, Korytowski MT, Kosiborod M, Maynard GA, Montori JJ, Van den Berghe G. Management of hyperglycemia in hospitalized patients in non-critical care setting: an endocrine society clinical practice guideline. J Clin Endocrinol Metab. 2012;97(1):16-38. doi:10.1210/jc.2011-2098.

21. Moghissi ES, Korytkowski MT, DiNardo M, Einhorn D, Hellman R, Hirsch IB, Inzucchi SE, Ismail-Beigi F, Kirkman MS, Umpierrez GE. American Association of Clinical Endocrinologists and American Diabetes Association consensus statement on inpatient glycemic control. Diabetes Care J. 2009;32(6):1119-31.

22. Pelletier EM, Smith PJ, Boye KS, Misurski DA, Tunis SL, Minshall ME. Direct medical costs for type 2 diabetes mellitus complications in the US commercial payer setting: a resource for economic research. Appl Health Econ Health Policy. 2008;6:103-12. doi:10.1007/BF03256126.

23. Department of Health and Human Services. How to use the Medicare National Correct Coding Initiative (NCCI) tool. https://www.cms.gov/Outreach-and-Education/Medicare-LearningNetwork-MLN/MLNProducts/Downloads/How-To-Use-NCCITools.pdf. Accessed 26 June 2016.

24. American Heart Association. Classification of functional capacity and objective assessment. http://my.americanheart.org/professional/ StatementsGuidelines/ByPublicationDate/PreviousYears/Classification-of-Functional-Capacity-and-Objective-Assessment_UCM_423 811_Article.jsp\#.VjzWp_Mo6JA. Accessed 23 July 2015.

25. American Hospital Association ${ }^{\circledR}$. Hospital billing explained. http://www.aha.org/content/13/hospbill-explain.pdf. Accessed 26 June 2016.

26. Haddix AC, Teutsch SM, Corso PS. Prevention effectiveness: a guide to decision analysis and economic evaluation. New York: Oxford University Press; 2003.

27. Research Data Assistance Center. Calculating "cost": cost-to charge ratios. http://www.resdac.org/calculating-cost-cost-tocharge-ratios.html. Accessed 23 July 2015.

28. US Department of Labor; Bureau of Labor Statistics. CPI inflation calculator. http://www.bls.gov/data/inflation_calculator.htm. Accessed 23 July 2015.

29. Powers B, Jain SH, Cutler D, Obermeyer Z. Pysician-level parctice variation: who you see is what you get. Health Affairs Blog. 23 September 2015. http://healthaffairs.org/blog/2015/09/ 23/physician-level-practice-variation-who-you-see-is-what-you-get/. Accessed 23 July 2015.

30. Griswold M, Parmigiani G, Potosky A, Lipscomb J. Analyzing health care costs: a comparison of statistical methods motivated 
by Medicare colorectal cancer charges. Biostatistics. 2006;1(1):1-23. http://www.biostat.jhsph.edu/project/seermed/ paper/pap033104.pdf. Accessed July 23, 2015.

31. SAS. SAS/STAT ${ }^{\circledR} 9.2$ User's Guide. The GENMOD Procedure (Book Excerpt). http://support.sas.com/documentation/cdl/en/ statug/66103/HTML/default/viewer.htm\#statug_genmod_overview. htm. Accessed 23 July 2015.

32. IBM Corp. IBM SPSS Statistics for Windows, Version 22.0. Armonk, NY: IBM Corporation; 2013.
33. Taheri PA, Butz DA, Greenfield LJ. Length of stay has minimal impact on the cost of hospital admission. J Am Coll Surg. 2000;191(2):123-30.

34. http://kff.org/other/state-indicator/expenses-per-inpatient-day/? currentTimeframe $=0 \&$ sortModel $=\% 7 \mathrm{~B} \% 22$ colld $\% 22: \% 22$ Location $\% 22, \% 22$ sort $\% 22: \% 22$ asc $\% 22 \% 7 \mathrm{D}$ 